\title{
Identification of potential microRNA-target pairs associated with osteopetrosis by deep sequencing, ITRAQ proteomics and bioinformatics
}

\author{
Minglin $\mathrm{Ou}^{1}$, Xiaoqing Zhang ${ }^{1}$, Yong $\mathrm{Dai}^{1}$, Jieying Gao ${ }^{1}$, Mingsong Zhu ${ }^{1}$, Xiangchun Yang ${ }^{1}$, Yuchao $\mathrm{Li}^{1}$, \\ Ting Yang ${ }^{1}$ and Min Ding ${ }^{\star, 1}$
}

MicroRNAs aberrantly express in many human diseases including some metabolic bone disorders. They have been found to be associated with osteoclast differentiation and function, which makes them attractive candidates for the therapy of bone. However, the potential clinical application of microRNAs in therapeutics rests heavily upon our in-depth understanding of microRNAs and their targets. To identify potential microRNA-target pairs associated with osteopetrosis, we performed a system approach including deep sequencing, iTRAQ quantitative proteomics, and bioinformatics in the peripheral blood mononuclear cells (PBMCs) taken from patients with osteopetrosis and health donors. Notably, 123 differently expressed microRNAs, 173 differently expressed proteins, and 117 computationally predicted microRNA-target pairs with reciprocally expressed level in PBMCs were found in the two sample groups. Functional annotation identified that the microRNA-target pairs were involved in cell growth, differentiation, cellular signaling network, and the network highlighted the microRNA-target pair of has-miR-320a and ADP ribosylation factor 1 (Arf1) potentially associated with CLCN7 mutations in osteopetrosis. The pair of has-miR-320a and Arf1 was further verified by real-time PCR, western blot, and the interaction between has-miR-320a and its targeted sequence on the Arf 1 mRNAs was confirmed by luciferase assay. Collectively, the present study established a new system approach for the investigation of microRNAs, and the microRNA-target pairs, particular has-miR-320a and Arf1, may have important roles in osteopetrosis.

European Journal of Human Genetics (2014) 22, 625-632; doi:10.1038/ejhg.2013.221; published online 2 October 2013

Keywords: micorRNA profile; protein profile; osteopetrosis

\section{INTRODUCTION}

MicroRNAs (miRNAs) are a class of single-stranded small noncoding RNAs that are 19-25 nucleotides (nt) in length, which regulate about $50 \%$ of human genes and has been hailed as one of the most important breakthroughs in biology. ${ }^{1}$ They are found to be aberrantly expressed in many human diseases including bone disorders. Recently, some researchers have indicated that miRNAs were associated with osteoclast differentiation, function, and impaired osteoclastogenesis, and could be found by miRNA silencing, which are make them attractive candidates for the therapy of some bone disorders. ${ }^{2}$ Obviously, the potential clinical application of microRNAs in therapeutics rests heavily upon our in-depth understanding of miRNAs and their targets under certain pathological conditions. Osteopetrosis is a disorder of abnormal bone metabolism, which may be caused by osteoclast failure and characterized by increased bone density on radiographs. It may be an inherited polygenic disorder, heterozygous mutations in CLCN7 may be one important cause, and other genes or regulators may also be involved in the pathological process of osteopetrosis. ${ }^{3}$ However, the roles of miRNAs in osteopetrosis remain to be further researched till now.

miRNAs play their biological roles by binding to some complementary sites of their targeted mRNA in $3^{\prime}$-untranslated regions by unknown mechanisms, and they are found to have some key transacting factors that regulate gene expression in posttranscription. ${ }^{1}$ Some bioinformatic algorithms, such as TargetScan, PicTar, and miRanda, have been constructed for miRNA target prediction now. However, all of these algorithms predict a great number of potential targets, which could not all be experimentally validated. ${ }^{4}$ On the other hand, system approaches with integrative miRNA and gene expression data may be able to identify higher confidence candidates, and fewer false-positive candidates. Considering that miRNA regulated target gene expression only at the protein and not at the mRNA level, it is reasonable to hypothesize that reciprocal expression of an miRNA and a computational algorithm predicted target protein under one particular physiological condition would be more valuable to identify diseasespecific miRNA-target pairs.

Peripheral blood mononuclear cells (PBMCs) have been proved to be valuable for understanding osteoclast biology and the pathological process of osteopetrosis, because osteoclasts are derived from hemopoietic precursor cells, including cells in the peripheral blood. ${ }^{5}$ Recent research indicates that manipulation of miRNA can control osteoclastogenesis in PBMCs culture systems, and miRNAs may involve in the generation of osteoclasts by regulating their targets in

\footnotetext{
${ }^{1}$ Key Laboratory of Laboratory Medical Diagnostics, Ministry of Education, Chongqing Medical University, Chongqing, China

*Correspondence: Professor M Ding, Key Laboratory of Laboratory Medical Diagnostics, Ministry of Education, Chongqing Medical University, Chongqing 400016, China. Tel: +86 02368485239; Fax: +86 02368485992; E-mail: dingmin@cqmu.edu.cn
}

Received 4 April 2013; revised 24 August 2013; accepted 28 August 2013; published online 2 October 2013 
PBMCs. ${ }^{6}$ Therefore, identification of potential miRNA-target pairs in PBMCs may be essential for the understanding of miRNA-based regulation in osteopetrosis. Given a negative relationship of the expression patterns between miRNAs and their target proteins, we combined deep-sequencing, isobaric tag for relative and absolute quantification technique and liquid chromatography tandem mass spectrometry (iTRAQ LC-MS/MS), and bioinformatics together to identify miRNA-target pairs in PBMCs taken from osteopetrosis patients.

\section{MATERIALS AND METHODS}

\section{Human samples}

Two groups of samples (osteopetrosis and control) were collected, and written informed consents were obtained from every patient and health donor. Blood samples were obtained from six patients of osteopetrosis with mutations in CLCN7 (rs387907576: A>G (RefSeq NM_001287.5)), and nine from age- and sex- matched healthy donors. The diagnosis of osteopetrosis was based on genetic diagnosis, typical clinical, hematological parameters, and radiographs of the spine and of the pelvis; and the osteopetrosis patients involved in this study have been descripted in our previous study. ${ }^{7}$ Approximately $5 \mathrm{ml}$ heparinized venous blood sample was taken from each subject; PBMCs in each blood sample were separated using Ficoll-Paque (GE Healthcare Life Sciences, Piscataway, NJ, USA). From each donation we created two aliquots, one was used for RNA extraction using TRIzol Reagent (Invitrogen, Carlsbad, CA, USA) and the other one was used for peptide and protein identification. All of the samples were stored at $-80^{\circ} \mathrm{C}$ until further analysis.

\section{Deep sequencing}

RNA integrity was checked by an Agilent 2100 Bioanalyzer (Agilent Technologies, Santa Clara, CA, USA). For the six samples in the osteopetrosis group and the nine samples in the control group, aliquots of total RNA from each subject were pooled, respectively, and subjected to osteopetrosis library and control library construction. The two libraries were sequenced by Solexa sequencing technology platform (BGI, Shenzhen, China), respectively. The procedures of library construction and sequencing were described in our previous research. ${ }^{8}$ Briefly, small RNA fragments (18-30 nt) were isolated from total RNA samples by PAGE gel, and then ligated $5^{\prime}$ and $3^{\prime}$ RNA adaptors. After purification, the products were reverse transcribed, amplified, and sequenced. The sequence tags (50 nt) from sequencing were gone through data cleaning first, which includes getting rid of the low-quality tags and several kinds of contaminants from the $50 \mathrm{nt}$ tags. All of high-quality clean reads between 18 and $30 \mathrm{nt}$ were aligned to the human reference genome (hg19) using SOAP program for expression and distribution analysis on the genome. Small RNA tags were then aligned to the miRNA precursor, mature miRNA in miRBase18. Novel miRNA candidates were predicted using Mireap by exploring their secondary structures, and also the minimum free energy of the unannotated small RNA tags. Differential expression of miRNA between osteopetrosis and control library were calculated by relative expression analysis.

\footnotetext{
Measurement of individual miRNA using real-time PCR

Expression levels of miRNA of each pooled sample analyzed by deep sequencing (osteopetrosis group, $n=6$; control group, $n=9$ ) were quantified using real-time PCR and the procedures was previously described in research with a minor modification. ${ }^{9}$ In brief, the extracted total RNA was treated with RNase-free DNAase I. Small RNAs were then reverse transcribed to cDNA using miRNA-specific stem-loop-like RT primer and amplified by the ABI PRISM 7500 Sequence Detection System (Applied Biosystems, Foster, CA, USA). U6 small nuclear RNA was used as an internal normalizer. Ct values were converted to fold expression changes by $2^{-\triangle \triangle \mathrm{CT}}$ method. The primers used for the amplification of the hsa-miR-320a and U6 were as follows: hsa-miR-320a, $5^{\prime}$-ACACTCCAGCTGGGAAAAGCTGGGTTGAGAGGGCG-3' (forward), $5^{\prime}$-CTCAACTGGTGTCGTGGA-3' (reverse); U6, 5' -CTCGCTTCG GCAGCACA-3' (forward), 5'-AACGCTTCACGAATTTGCGT-3' (reverse).
}

\section{ITRAQ LC-MS/MS analysis}

The process of protein extraction and iTRAQ sample labeling were performed as described by our previous research with minor modifications according to Qiao et al. ${ }^{10,11}$ Briefly, for the six samples in the osteopetrosis group and the nine samples in the control group, aliquots of PBMCs protein from each subject were pooled respectively. One hundred micrograms of protein from each corresponding group pool protein was digested with trypsin, and labeled according to the iTRAQ protocol (Applied Biosystems). The labeled digests were subjected to LC-MS/MS analysis. The MS data were processed by Proteome Discoverer software (version 1.2.0.208) (Thermo Scientific, Bremen, Germany) and performed protein identification and quantification using mascot 2.3.02 (Matrix Science, London, UK) against the database of International Protein Index (IPI, human version 3.87) (see Supplementary Material for details).

\section{Western blot}

Protein abundance of the pooled samples previously analyzed by iTRAQ LC-MS/MS was confirmed essentially as previously described using western blot. ${ }^{12}$ Total protein samples were added in electrophoretic buffer containing $\beta$-mercaptoethanol before SDS-PAGE. GAPDH (Kangcheng, Shanghai, China) was used as a loading control. The primary antibody for ADP-ribosylation factor 1 (Arfl) was obtained from Abcam (Abcam Inc., Cambidge, MA, USA). And the peroxidase-conjugated goat anti-rabbit IgG secondary antibody was obtained from SouthernBiotech (Birmingham, AL, USA). The band intensity of western blot analysis was repeated three times using Image $\mathrm{J}$ software (National Institutes of Health, Bethesda, MD, USA).

\section{Bioinformatic analysis of miRNA-target pairs}

Targets of differentially expressed miRNAs were predicted using starBase program, which compiles prediction results from TargetScan, PicTar, RNA22, PITA, and miRanda. ${ }^{13}$ Standard human gene symbols of differentially expressed proteins found by iTRAQ LC-MS/MS analysis were used to search the list of miRNA targeted genes. The proportion of inversely expressed miRNA and protein pairs that were computationally predicted $\left(P_{\mathrm{i}}\right)$, was calculated as $P_{\mathrm{c}}=n_{\mathrm{i}} / N_{\mathrm{i}}$, where $n_{\mathrm{i}}$ was the number of inversely expressed miRNA and protein pairs that were computationally predicted and $N_{\mathrm{i}}$ was the total number of computationally predicted miRNA and protein pairs; and eight miRNAs were randomly selected from the 411 miRNAs found by deep sequencing, ${ }^{14}$ the proportion of inversely expressed miRNA and protein pairs that were randomly selected $\left(P_{\mathrm{m} 1}\right)$ was calculated. Similarly, the proportion of computationally predicted miRNA and protein pairs that were inversely expressed $\left(P_{\mathrm{c}}\right)$, and the proportion of computationally predicted miRNA and protein pairs that were randomly selected $\left(P_{\mathrm{m} 2}\right)$ were calculated (see Supplementary Material for the details of calculations). $\chi^{2}$ Tests were performed to examine if $P_{\mathrm{i}}$ was significantly different than that of $P_{\mathrm{ml}}$, and also $P_{\mathrm{c}}$ was significantly different than that of $P_{\mathrm{m} 2}$.

Candidate miRNA-target pairs were collected and further analyzed. GO and KEGG pathway mapping were performed by web-accessible DAVID (version 6.7) annotation system. Potential inter-relationship of ClC-7, the miRNA targets, and the differently expressed proteins were analyzed using KEGGSOAP, MIPS database, and co-citation calculation in PubMed; finally, a network was constructed by integrating the results of the above three types of data after comprehensive considerations. ${ }^{15}$

\section{Cell culture, transfection, and luciferase assays}

The sequence (chr1:228285715-228286913, $1199 \mathrm{bp}$ ) from the 3'-UTR of ARF1 (NM_001024226.1) and its mutant sequence were amplified and cloned into psi-CHECK2 reporters (Promega, Madison, WI, USA) by Land (Guangzhou, China). HEK293 T cells were transfected in 24-well plates with or without $0.5 \mu \mathrm{g}$ of ARF1 $3^{\prime}$-UTR luciferase reporter plasmid or its mutant, and cotransfected with the hsa-miR-320a mimic ( $50 \mathrm{~nm}$ ) or nonspecific miRNA mimic negative control (50 or $100 \mathrm{~nm}$ ) or hsa-miR-320a inhibitor (100 nM) (RiboBio Co., Guangzhou, China) per well, using Lipofectamine RNAiMAX (Invitrogen). The cultures were harvested and analyzed by western bolt or the Dual-Glo Luciferase Assay System (Promega) after $48 \mathrm{~h}$, and the activity of Firefly luciferase was normalized to the activity of Renilla luciferase as a control 
for transfection efficiency. ${ }^{16}$ All of these experiments were repeated two to three times.

\section{Statistics}

Data were analyzed using Student's $t$-test by SPSS, except some special cases that were described above. $P<0.05$ was considered as significant.

\section{RESULTS}

\section{miRNA expression profile}

Deep sequencing data was deposited in Sequence Read Archive (http://www.ncbi.nlm.nih.gov/sra) under accession no. PRJNA210360. In the two small RNA libraries, we obtained total 20674132 small RNA reads after filtering of the high-quality clean reads. The expression profile showed that a large set of miRNAs $(20,674,132)$ were expressed in both libraries, a small set (192, 997/20, 674, 132, $0.93 \%$ ) was not expressed, whereas 153, 045/20, 674, $132(0.74 \%)$ miRNAs were expressed only in the osteopetrosis library. Compared with the control group of known miRNAs, we found that 63 miRNAs were expressed at a significantly higher level, 288 miRNAs were expressed at approximately equal levels, and 60 miRNAs were expressed at significantly reduced levels in osteopetrosis library (Supplementary Table S1). On the basis of the sequences that could not be found in the miRBase18, we identified 96 novel miRNAs in the osteopetrosis library (Supplementary Table S2). Hsa-miR-320a was further analyzed by real-time PCR (Figure 1a). Compared with deep sequencing, real-time PCR analysis indicated similar relative expressed level of the hsa-miR-320a.

\section{Protein expression profile}

The iTRAQ-LC-MS/MS data was deposited in ProteomeXchange Consortium (http://proteomecentral.proteomexchange.org) with identifier PXD000346. A total of 3574 unique peptides were detected and led to the identification of 1227 proteins with a mascot score of at least 20. Relative quantification of proteins was based on the ratio of peak areas from MS/MS spectra, and the $\mathrm{m} / \mathrm{z}$ of 114 (osteopetrosis) and 115 (control) were involved in this research. Compared with the control group, we found 173 differently expressed proteins totally, including 78 upregulated and 95 downregulated expression proteins, giving tryptic peptides with 1.5 -fold or more change and a $P$-value $<0.05$ in the osteopetrosis group (Supplementary Table S3). The differential expression of Arf1 between the osteopetrosis and control group was verified by western blot (Figures $1 b$ and $c$ ), which indicated a similar relative expressed level compared with iTRAQ LC-MS/MS analysis.

\section{Identification of miRNA-targets pairs}

Totally, 117 of 10665 reciprocally expressed miRNA and protein pairs were computationally predicted to be miRNA-target pairs (Supplementary Table S4), 14 pairs were predicted by at least three algorithms (Table 1), and three miRNA-target pairs, including miR-424/CALU, miR-223/HSP90B1, and miR-21/RTN4 had been
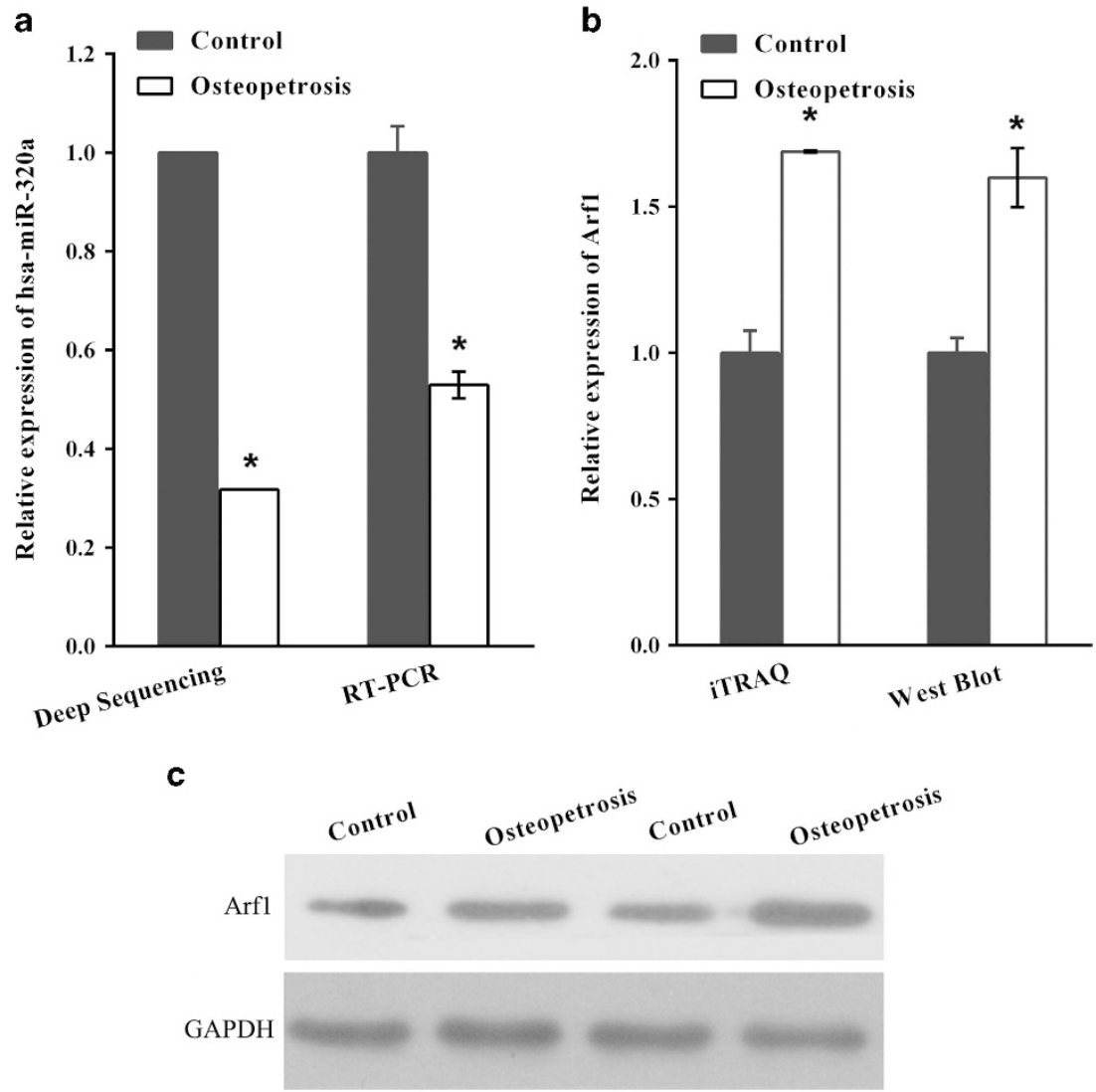

Figure 1 The relative expression level of the pairs of has-miR-320a and Arf1 in the PBMCs sample group of control and osteopetrosis. (a) Has-miR-320a expression level analyzed by deep sequencing ( $P$-values were calculated by relative expression analysis), and real-time PCR. (b and $\mathbf{c}$ ) Arf1 expression level measured by iTRAQ LC-MS/MS and western blots. With $\left(^{*}\right)$ significantly different from the other group. 
reported in the literature. ${ }^{17-19}$ The proportion of inversely expressed miRNA and protein pairs that were computationally predicted $\left(P_{\mathrm{i}}=0.4680\right)$ was significantly greater than what can be expected from random selected $\left(P_{\mathrm{m} 1}=0.1814, P<0.01\right)$, which indicated that the miRNA-target interactions predicted by online algorithms were more likely to be inversely correlated than by chance (Figure 2a), and the proportion of computationally predicted miRNA-target pairs that were inversely expressed $\left(P_{\mathrm{c}}=0.0110\right)$ was also significantly greater than what can be expected from random selected $\left(P_{\mathrm{m} 2}=0.0036\right.$, $P<0.05)$, which indicated that the inversely expressed miRNAs and proteins were more likely to be predicted as miRNA-target pairs by online algorithms than by chance (Figure $2 \mathrm{~b}$ ). The fold changes of the miRNA-target pairs (in Supplementary Data and Supplementary Table S4) predicted by at least three algorithms were not significantly different from the pairs predicted by only one algorithm (absolute $\log 2$ ratio, $2.61 \pm 0.12$ vs $2.70 \pm 0.07, P=0.593$ ). It indicated that there was no positive correlation between the fold changes of the miRNA-target pairs and the number of predicted online algorithms in this research. The genes of proteins potentially regulated by differently expressed miRNAs produced a total of 46 GO terms

Table 1 Reciprocally expressed miRNAs and computationally predicted targets

\begin{tabular}{|c|c|c|c|c|c|c|c|c|c|}
\hline \multirow[b]{2}{*}{$\operatorname{miRNA}$} & \multirow[b]{2}{*}{ miRNA $\log 2$ ratio } & \multirow[b]{2}{*}{ Target symbol } & \multirow[b]{2}{*}{ Protein } & \multirow[b]{2}{*}{ Protein $\log 2$ ratio } & \multicolumn{5}{|c|}{ starBase } \\
\hline & & & & & TargetScan & PicTar & RNA22 & PITA & miRanda \\
\hline hsa-miR-122(5p) & 2.28 & CALM3 & Calmodulin & -0.78 & Yes & Yes & & Yes & Yes \\
\hline hsa-miR-130b(3p) & 1.06 & CMPK1 & UMP-CMP kinase isoform a & -1.11 & Yes & Yes & & Yes & Yes \\
\hline hsa-miR-15b(5p) & 1.97 & CALU & Isoform 2 of calumenin & -0.68 & Yes & & Yes & Yes & Yes \\
\hline hsa-miR-15b(5p) & 1.97 & CMPK1 & UMP-CMP kinase isoform a & -1.11 & Yes & Yes & Yes & & \\
\hline hsa-miR-15b(5p) & 1.97 & $R A D 23 B$ & UV excision repair protein RAD23 homolog B & -1.33 & Yes & Yes & & Yes & Yes \\
\hline hsa-miR-16(5p) & 1.34 & CALU & Isoform 2 of calumenin & -0.68 & Yes & & Yes & Yes & Yes \\
\hline hsa-miR-16(5p) & 1.34 & $R A D 23 B$ & UV excision repair protein RAD23 homolog B & -1.33 & Yes & Yes & Yes & Yes & Yes \\
\hline hsa-miR-424(5p) & 1.86 & $C A L U$ & Isoform 2 of calumenin & -0.68 & Yes & & & Yes & Yes \\
\hline hsa-miR-424(5p) & 1.86 & $R A D 23 B$ & UV excision repair protein RAD23 homolog B & -1.33 & Yes & & & Yes & Yes \\
\hline hsa-miR-148b(5p) & -1.30 & RTN4 & Isoform 3 of reticulon-4 & 1.45 & & & Yes & Yes & Yes \\
\hline hsa-miR-148b(5p) & -1.30 & HSP9OB1 & Endoplasmin precursor & 0.70 & & Yes & & Yes & Yes \\
\hline hsa-miR-152 & -1.24 & RTN4 & Isoform 3 of reticulon-4 & 1.45 & & & Yes & Yes & Yes \\
\hline hsa-miR-152 & -1.24 & HSP9OB1 & Endoplasmin precursor & 0.70 & & Yes & & Yes & Yes \\
\hline hsa-miR-320a & -1.66 & ARF1 & ADP-ribosylation factor 1 & 0.80 & Yes & Yes & Yes & Yes & Yes \\
\hline
\end{tabular}

miRNA log 2 ratio, fold-change (logb2 osteopetrosis/control) analyzed by miRNA sequencing; Target gene symbol, gene symbol of miRNA target; protein log 2 ratio, fold-change (log 2 osteopetrosis/control) analyzed by iTRAQ quantitative proteomic; 'yes', indicates that the target has one or more predicted binding sites for the miRNA. Only the pairs predicted by at least three algorithms are listed.
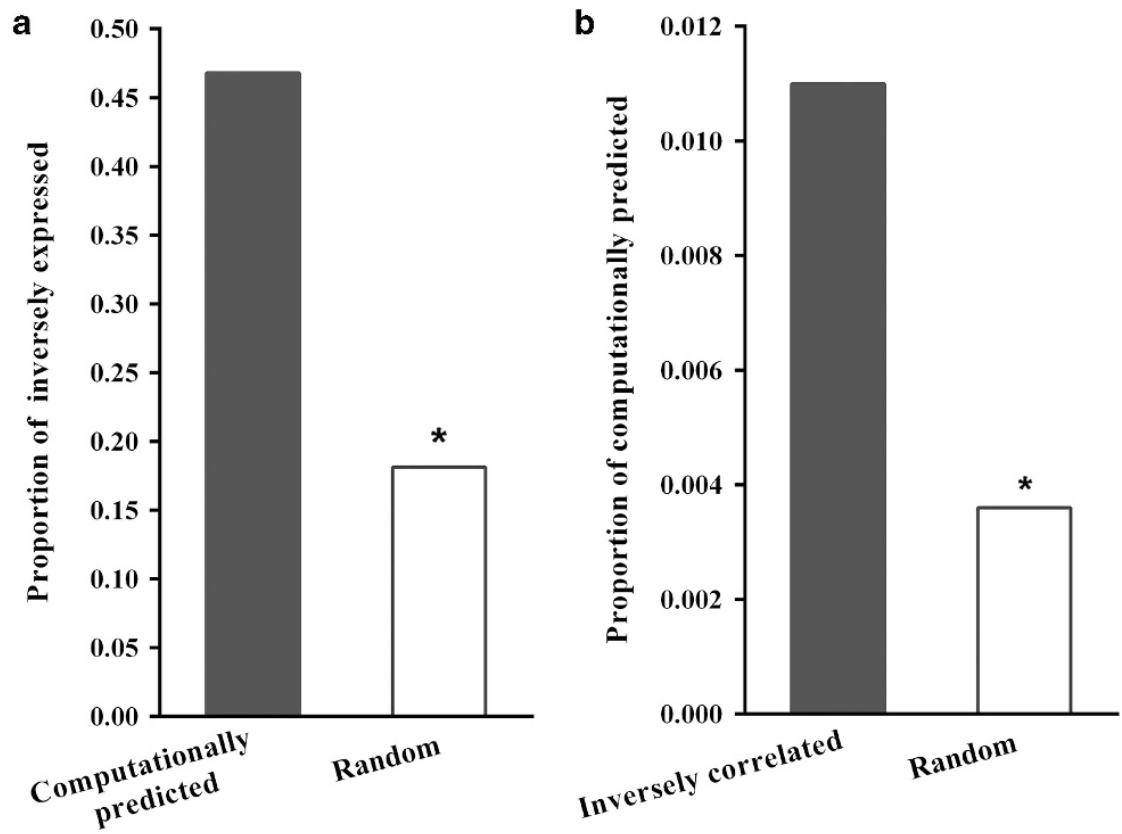

Figure 2 Analysis of computationally predicted miRNA target pairs and reciprocal expression of miRNA and proteins pairs. (a) The proportion of inversely expressed miRNA and protein pairs that were computationally predicted or randomly selected. (b) The proportion of computationally predicted miRNA and protein pairs that were inversely expressed or randomly selected. Details of the calculation are described in the Materials and methods section, with $\left({ }^{*}\right)$ significantly different from the other group ( $\chi^{2}$ tests). 
(Supplementary Table S5) and two KEGG pathways, such as 'Vascular smooth muscle contraction' and 'Neurotrophin signaling pathway', were significantly enriched $(P<0.05)$; the potential network indicated that the ClC-7, the miRNA targets, and the differently expressed proteins were connected with each other directly or indirectly (Supplementary Figure S1), and there was a direct relationship of co-citation between CLCN7 and ARF1, SLC9A1. One of the predicted miRNA-target pairs, has-miR-320a and Arf1, was chosen to be further tested in vitro. The band intensity of western blot of Arf1 in the culture with has-miR-320a mimics was significantly lower compared with the control and nonspecific miRNA negative control (Figures $3 \mathrm{a}$ and $\mathrm{b}$ ), which indicated that Arf1 expression may be inhibited by has-miR-320a, and the band intensity of western blot of Arfl in the culture with has-miR-320a inhibitor was significantly higher compared with the control and nonspecific miRNA negative control (Figures $3 \mathrm{c}$ and $\mathrm{d}$ ), which indicated that has-miR-320a inhibitor significantly increased the protein expression level of Arf1. Luciferase assays found that luciferase reporter gene expression were effectively inhibited by has-miR-320a mimics in the culture supplied with plasmid containing the $3^{\prime}$-UTR of $A R F 1$, but the luciferase reporter gene expression were not inhibited by has-miR-320a mimics in the culture supplied with plasmid containing the $3^{\prime}$-UTR of ARF1 mutant, which indicated that $3^{\prime}$-UTR of ARF1 may be one specific binding site of has-miR-320a (Figure 4).

\section{DISCUSSION}

In this research, we tried to identify miRNA profile, protein profile and reveal potential miRNA-target pairs in PBMCs of a typical disorder of abnormal bone metabolism by a novel and combinatorial approach involving deep-sequencing, iTRAQ quantitative proteomic, and bioinformatic analysis.

Deep sequencing generated more than 10 million small RNA sequence reads in this study. Sequence tags analysis show that about one-third of miRNAs were expressed at significantly different level between osteopetrosis and health donors. In the known miRNAs with significantly increased expression level, one member of the highly conserved miR-23abc/23b-3p family (hsa-miR-23a) is the most abundant. Hsa-miR-23a is among several miRNAs currently a

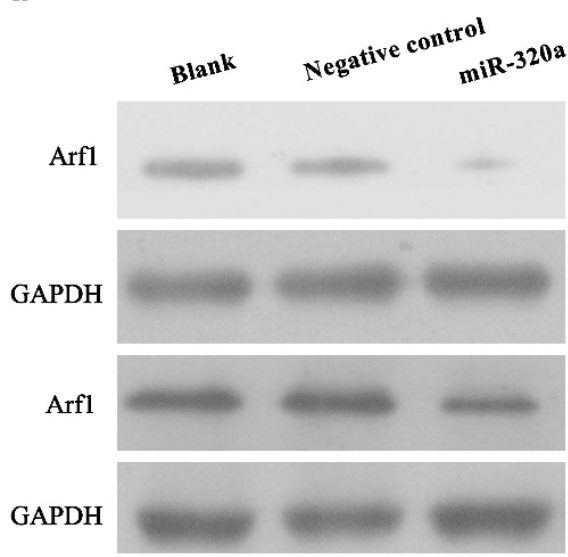

C

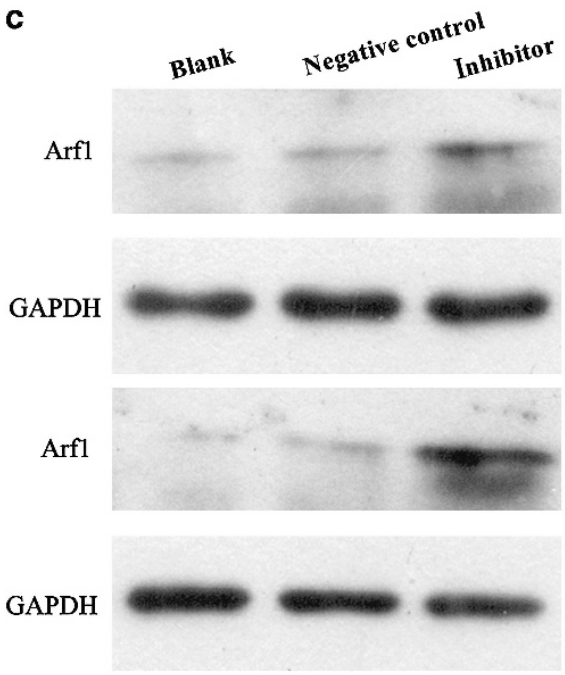

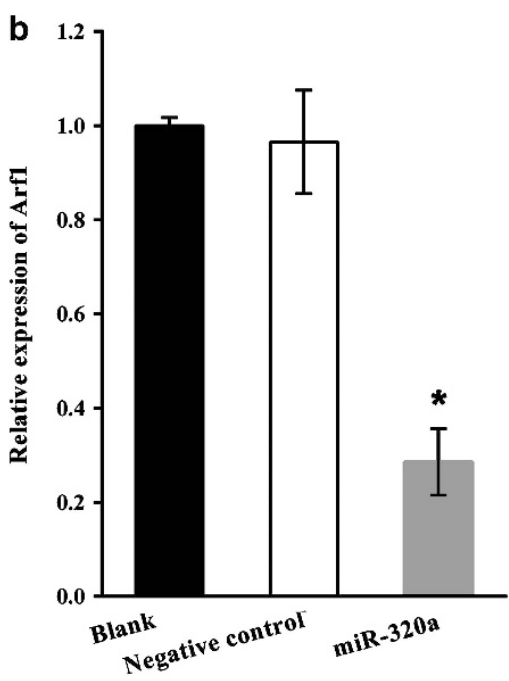

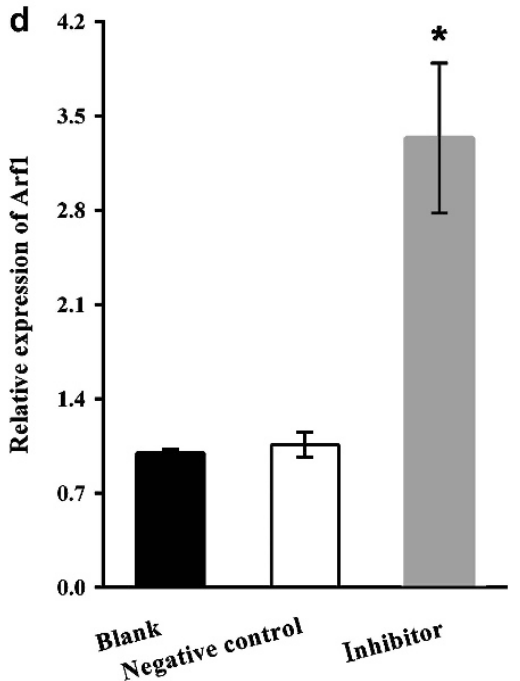

Figure 3 The relative expression level of Arf1 in the 293 T-cell cultures. (a and $\mathbf{b}$ ) In the culture of 293 T cells, compared with the control without miRNA mimic and negative control with nonspecific miRNA mimic ( $50 \mathrm{~nm})$, has-miR-320a mimic ( $50 \mathrm{~nm}$ ) significantly decreased the protein expression level of Arf1. (c and d) Compared with the control without miRNA mimic and negative control with nonspecific miRNA mimic (100 nm), has-miR-320a inhibitors (100 nn) significantly increased the protein expression level of Arf1. These experiments were repeated two times, with $\left({ }^{*}\right)$ significantly different from the other group. 


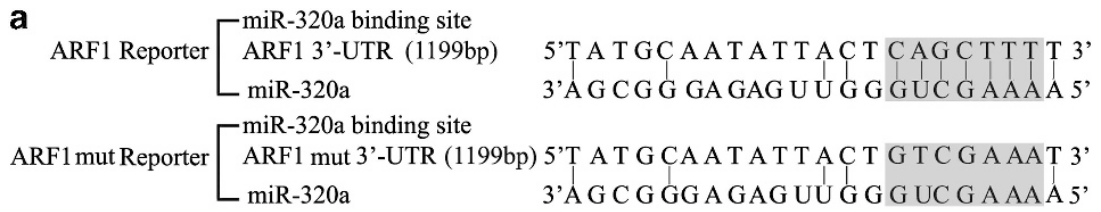

b

ARF1 Reporter $\frac{\mathrm{T} 7}{\text { Promoter }}$ Renilla
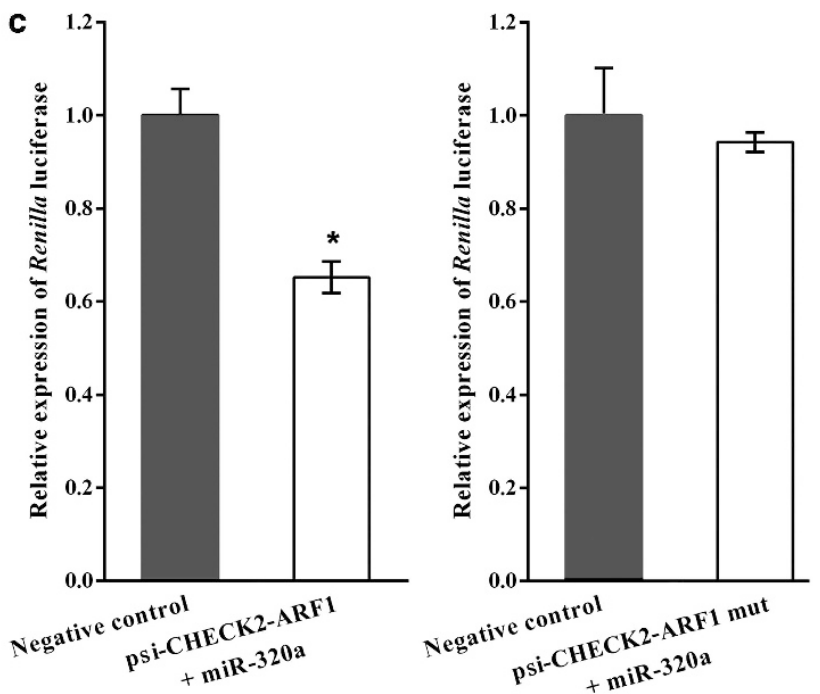

Figure 4 Confirmation of the interaction between has-miR-320a and its targeted sequence on Arf1 mRNA. (a) The representative sequence is the complementary sites of ARF1 and has-miR-320a. In gray background is the 'seed' region that is complementary to has-miR-320a, or the mutant site. (b) Schematic representation of the reporters used in the luciferase assay: a 1199-bp (ARF1 reporter) region of the 3'-UTR of human ARF1 containing the putative has-miR-320a target sites (in gray background), or a 1199-bp ( ARF1 mut reporter) region of the 3'-UTR of human ARF1 mutant without the putative has-miR-320a target sites were cloned into the psi-CHECK2. (c) Luciferase assays of ARF1 reporters and ARF1 mut reporters. In the negative control of 293 T cells transfected with wild-type psi-CHECK2-ARF1 (ARF1 reporter, $0.5 \mu \mathrm{g}$ ) was co-transfected with nonspecific miRNA mimics (50nM), and the other groups of 293 T cells transfected with ARF1 reporter $(0.5 \mu \mathrm{g})$ was co-transfected with has-miR-320a mimics ( $50 \mathrm{~nm})$. In the negative control of 293 T cells transfected with mutant-type psi-CHECK2-ARF1 (ARF1 mut reporter, $0.5 \mu \mathrm{g}$ ) was co-transfected with nonspecific miRNA mimics (50 nm), and the other groups of $293 \mathrm{~T}$ cells transfected with ARF1 mut reporter $(0.5 \mu \mathrm{g})$ were co-transfected with has-miR-320a mimics (50 nm). These experiments were repeated three times, with $\left({ }^{*}\right)$ significantly different from the other groups.

considered as suppressors of osteoblast differentiation, upregulated hsa-miR-23a in PBMCs may be involved in the abnormal osteoclasts found in osteopetrosis-specific PBMC-induced osteoclasts. $^{20}$ Hsa-miR-29b-3p in the miR-29abcd family was among the most significantly downregulated miRNAs in osteopetrosis library. This is consistent with a putative role of downregulation of hsa-miR-29b-3p in decreased osteoclastogenesis in osteopetrosis-specific PBMCinduced osteoclasts. Further, the biological functions of has-miR$29 \mathrm{~b}$ have been found to contribute to the positive regulation of osteoblast differentiation. ${ }^{21}$ Taken together, these findings suggest that known miRNAs with significantly differently expressed level have putative key roles in osteoblast differentiation in osteopetrosis specific PBMC-induced osteoclasts. In addition, we predicted 96 novel miRNAs in osteopetrosis library, based on the characteristic stemloop hairpin secondary structure in miRNA precursors. Importantly, most novel miRNAs were expressed at a low level compared with previously known miRNAs, indicating that deep sequencing is highly sensitive and able to detect novel miRNAs.

In parallel to the miRNA expression profiling, we also have described the changes of protein profile using iTRAQ LC-MS/MS.
This technology is able to mix the samples labeled with iTRAQ, and then perform subsequent processing and isotope dilution mass analysis under identical condition. Therefore, mass tagging of peptides ensures any differences in the protein expression levels are because of initial differences in the samples, and not an artifact in the sample handling or processing. ${ }^{22}$ As a result, we revealed a large-scale proteomic changes in osteopetrosis. One protein, such as RhoA (IPI01015923), has been implicated to be involved in osteopetrosis. ${ }^{23}$ RhoA is one of the best-known members of the Rho GTPases, and is required for fMet-Leu-Phe-stimulated TRAF family member associated NF- $\kappa \mathrm{B}$ activation; furthermore, NF- $\kappa \mathrm{B}$ activator is a negative regulator of osteoblast differentiation. ${ }^{24}$ Therefore, the differentiation of osteopetrosis-specific PBMC-induced osteoclasts may be suppressed by upregulated expression of RhoA. However, the magnitude of proteomic changes suggested possible involvement of other regulators.

On the other hand, miRNAs have critical roles in many human diseases. Other research has utilized transcriptomic-based approaches for identifying disease-specific miRNA targets. However, they suffer from limitations in miRNA decreased protein synthesis that are not 
susceptible to mRNA degradation would be missed. ${ }^{25}$ Recently, some researches based on microarray and label-free quantification method have been found to be valuable for the understanding of miRNAs and their target interactions; even though microarray technology suffers from limitations in sensitivity and specificity, ${ }^{26}$ and also the sensitivity of most proteomic analysis based on label-free quantification method by spectrometers is modulated by the nature of the processed samples. ${ }^{27}$ In this research, we improve the sensitivity of miRNA and protein identification by deep sequencing and iTRAQ quantitative proteomics respectively. A novelty of our research is that we identified more than one hundred potential miRNA-target pairs from computationally predicted targets, considering a negative relationship of the expression patterns between miRNA and their target protein is the most important parameter for accessing their interactions. Functional annotation indicated that they were involved in clusters of significantly relevant and meaningful biological processes. For example, GO terms related to molecular function, biological process, cellular component, and KEGG pathway related to neurotrophin signaling pathway that could be found in traf6-/ mice exhibited severe osteopetrosis, ${ }^{28}$ were highly represented and enriched in our study. Thus, although the exact mechanism remains unknown and requires further study, miRNAs may be involved in osteopetrosis by regulation of cell growth, differentiation, and cellular signaling network.

Our study identified the miRNA-target pair of has-miR-320a/Arf1 we chose for further analysis in vitro, partly because of it is predicted by TargetScan, PicTar, RNA22, PITA, and miRanda and partly because has-miR-320a is one of differently expressed miRNAs with the most abundant, and also because Arfs have been reported to overlap with the protein product of CLCN7 (ClC-7), ${ }^{29}$ and have important roles in osteoclast function and bone degradation. ${ }^{30}$ Arfs are a family of ubiquitously expressed Ras-like GTPases that have key roles in a great number of vesicular transport processes in eukaryotic cell. The Arfs family, which includes six members in humans, continuously cycle between the cytosol and different membranes, through GTP binding and hydrolysis, and are assisted by COP-related components involve in vesicular trafficking and organelle structure. ${ }^{31}$ Defects in Arfs have been found leading to abnormal membrane traffic and association with various human diseases. The translocation of cytosolic Arfs to membranes is driven by the acidic endosomal $\mathrm{pH}$ signaling, and defective Arfs trafficking may be as a result of deficient endosomal acidification due to inhibition of chloride channels. ${ }^{32}$ Arfl is an important member of the Arfs family, and it has been found colocalized with the late endosomal/lysosomal marker ClC-7. ${ }^{29}$ Interesting, our study identified that Arfl was upregulated expression in osteopetrosis PBMCs associated with CLCN7 mutation in this research. We hypothesize that in the PBMCs taken from osteopetrosis patients, downexpression of has-miR-320a, leads to the increased expression of Arf1, which may be a positive respond to the defective chloride channel, and may have roles in cellular functions, such as self-repair in vesicular trafficking. Obviously, a suitable cellular system research, for example, using target protector morpholinos that interfere with the pair of has-miR-320a and Arf1 by binding specifically to the has-miR-320a target sequence in the $3^{\prime}$-UTR in PBMCs culture systems is required to provide evidence of relative importance of this specific miRNA-target interaction in osteopetrosis. ${ }^{33}$

In conclusion, the present study establishes a new system approach for the identification of putative miRNAs involved in the pathological process of abnormal bone metabolism, and our data has mainly made three contributions in bone disorder research. Firstly, we have described a differential profile of miRNA expression in osteopetrotic PBMCs for the first time to the best of our knowledge. Secondly, we have greatly explored the research of osteopetrosis in proteomic profiling. Finally, we identified reciprocal expression of more than one hundred of the potential miRNA-target pairs, particularly hasmiR-320a/Arf1, which may be valuable for the understanding of miRNA biology and the development of new therapeutics in bone disease in the future.

\section{CONFLICT OF INTEREST}

The authors declare no conflict of interest.

\section{ACKNOWLEDGEMENTS}

We thankf JI Limnios and W Sui for their valuable help in this study. We also thank the PRIDE Team for their help in the submission of the data. This work was funded by Guangxi Key Laboratory of Metabolic Diseases Research (No. 12-071-32).

1 Krol J, Loedige I, Filipowicz W: The widespread regulation of microRNA biogenesis, function and decay. Nat Rev Genet 2010; 11: 597-610.

2 Kapinas K, Delany AM: MicroRNA biogenesis and regulation of bone remodeling. Arthritis Res Ther 2011; 13: 220.

3 Chu K, Koller DL, Snyder R et al: Analysis of variation in expression of autosomal dominant osteopetrosis type 2: searching for modifier genes. Bone 2005; 37: 655-661.

4 Iliopoulos D, Malizos KN, Oikonomou P, Tsezou A: Integrative microRNA and proteomic approaches identify novel osteoarthritis genes and their collaborative metabolic and inflammatory networks. PLoS One 2008; 3: e3740.

5 Boyle WJ, Simonet WS, Lacey DL: Osteoclast differentiation and activation. Nature 2003; 423: 337-342.

6 Shibuya H, Nakasa T, Adachi N et al: Overexpression of microRNA-223 in rheumatoid arthritis synovium controls osteoclast differentiation. Mod Rheumatol 2013; 23: $674-685$

7 Sui W, Ou M, Liang J et al: Rapid gene identification in a Chinese osteopetrosis family by whole exome sequencing. Gene 2013; 516: 311-315.

8 Sui W, Ou M, Chen J et al: MicroRNA expression profile of peripheral blood mononuclear cells of Klinefelter syndrome. Exp Ther Med 2012; 4: 825-831.

9 Unlu S, Tang S, na Wang E et al: Damage Associated Molecular Pattern MoleculeInduced microRNAs (DAMPmiRs) in human peripheral blood mononuclear cells. PLoS One 2012; 7: e38899.

10 Sui W, Tang D, Zou G et al: Differential proteomic analysis of renal tissue in lupus nephritis using ITRAQ reagent technology. Rheumatol Int 2011; 32: 3537-3543.

11 Qiao J, Wang J, Chen L et al: Quantitative iTRAQ LC-MS/MS proteomics reveals metabolic responses to biofuel ethanol in Cyanobacterial Synechocystis sp. PCC 6803. J Proteome Res 2012; 11: 5286-5300.

12 Nayak D: Sphingosine kinase 1 regulates the expression of proinflammatory cytokines and nitric oxide in activated microglia. Neuroscience 2010; 166: 132-144.

13 Yang JH, Li JH, Shao P, Zhou H, Chen YQ, Qu LH: starBase: a database for exploring microRNA-mRNA interaction maps from Argonaute CLIP-Seq and Degradome-Seq data. Nucleic Acids Res 2011; 39: D202-D209.

14 Tian Z, Greene AS, Pietrusz JL, Matus IR, Liang M: MicroRNA-target pairs in the rat kidney identified by microRNA microarray, proteomic, and bioinformatic analysis. Genome Res 2008; 18: 404-411.

15 Gao W, Xu J, Liu L, Shen H, Zeng H, Shu Y: A systematic-analysis of predicted miR-21 targets identifies a signature for lung cancer. Biomed Pharmacother 2012; 66: 21-28.

16 Rayner KJ, Esau CC, Hussain FN et al: Inhibition of miR-33a/b in non-human primates raises plasma HDL and lowers VLDL triglycerides. Nature 2011; 478: 404-407.

$17 \mathrm{Li} \mathrm{G}$, Cai M, Fu D et al: Heat shock protein 90B1 plays an oncogenic role and is a target of microRNA-223 in human osteosarcoma. Cell Physiol Biochem 2012; 30: 1481-1490.

18 Yang Y, Chaerkady R, Beer MA, Mendell JT, Pandey A: Identification of miR-z21 targets in breast cancer cells using a quantitative proteomic approach. Proteomics 2009; 9: 1374-1384.

19 Merlet E, Atassi F, Motiani RK et al: miR-424/322 regulates vascular smooth muscle cell phenotype and neointimal formation in the rat. Cardiovasc Res 2013; 98: $458-468$.

20 Kantaputra PN, Thawanaphong S, Issarangporn W et al: Long-term survival in infantile malignant autosomal recessive osteopetrosis secondary to homozygous p. Arg526Gln mutation in CLCN7. Am J Med Genet A 2012; 158A: 909-916.

$21 \mathrm{Li} \mathrm{Z}$, Hassan MQ, Jafferji M et al: Biological functions of miR-29b contribute to positive regulation of osteoblast differentiation. J Biol Chem 2009; 284: 15676-15684. 
22 Wang L, Dai Y, Qi S et al: Comparative proteome analysis of peripheral blood mononuclear cells in systemic lupus erythematosus with ITRAQ quantitative proteomics. Rheumatol Int 2012; 32: 585-593.

23 Del Fattore A, Cappariello A, Teti A: Genetics, pathogenesis and complications of osteopetrosis. Bone 2008; 42: 19-29.

24 Maruyama K, Kawagoe T, Kondo T, Akira S, Takeuchi O: TRAF family memberassociated NF- $\mathrm{KB}$ activator (TANK) is a negative regulator of osteoclastogenesis and bone formation. J Biol Chem 2012; 287: 29114-29124.

25 Großhans H, Filipowicz W: Proteomics joins the search for microRNA targets. Cell 2008; 134: 560-562.

26 Dhahbi JM, Atamna H, Boffelli D, Magis W, Spindler SR, Martin DIK: Deep sequencing reveals novel microRNAs and regulation of microRNA expression during cell senescence. PLoS One 2011; 6: e20509.

27 Nilsson T, Mann M, Aebersold R, Yates JR, Bairoch A, Bergeron JJM: Mass spectrometry in high-throughput proteomics: ready for the big time. Nat Methods 2010; 7: 681-685.
28 Yeiser EC, Rutkoski NJ, Naito A, Inoue J, Carter BD: Neurotrophin signaling through the p75 receptor is deficient in traf6 -/ - mice. J Neurosci 2004; 24: 10521-10529.

29 Wolff NA, Lee W-K, Thévenod F: Role of Arf1 in endosomal trafficking of protein-metal complexes and cadmium-metallothionein-1 toxicity in kidney proximal tubule cells. Toxicol Lett 2011; 203: 210-218.

30 Heckel T, Czupalla C, Santo AIE et al: Src-dependent repression of ARF6 is required to maintain podosome-rich sealing zones in bone-digesting osteoclasts. Proc Natl Acad Sci 2009; 106: 1451-1456.

31 D'Souza-Schorey C, Chavrier P: ARF proteins: roles in membrane traffic and beyond. Nat Rev Mol Cell Biol 2006; 7: 347-358.

32 Maranda B, Brown D, Bourgoin S et al: Intra-endosomal pH-sensitive recruitment of the Arf-nucleotide exchange factor ARNO and Arf6 from cytoplasm to proximal tubule endosomes. J Biol Chem 2001; 276: 18540-18550.

33 Staton AA, Giraldez AJ: Use of target protector morpholinos to analyze the physiological roles of specific miRNA-mRNA pairs in vivo. Nat Protocols 2011; 6: 2035-2049.

Supplementary Information accompanies this paper on European Journal of Human Genetics website (http://www.nature.com/ejhg) 